\title{
Oral Candida infection and colonization in solid organ transplant recipients
}

\author{
A. Dongari-Bagtzoglou ${ }^{1}$, P. Dwivedi ${ }^{1}$, E. loannidou ${ }^{1}$, M. Shaqman ${ }^{1}$, D. Hull ${ }^{2}$, and J. \\ Burleson $^{3}$ \\ 1 Department of Oral Health and Diagnostic Sciences, Division of Periodontology, School of Dental Medicine, \\ University of Connecticut Health Center, Farmington, CT, USA \\ 2Transplant Unit, Hartford Hospital, Hartford, CT, USA \\ 3Department of Community Medicine and Health Care, School of Medicine, University of Connecticut Health \\ Center, Farmington, CT, USA
}

\section{Abstract}

Introduction-Oral Candida carriage and infection have been reported to be associated with a greater risk for systemic infection in transplant recipients; however, a systematic analysis of the oral Candida titers and species has not been previously conducted. The objectives of this study were to determine the prevalence of oropharyngeal candidiasis, the oral carrier status, Candida titers and species in this population.

Methods-Ninety kidney and heart transplant subjects and 72 age-matched healthy controls were included. Swabs from the oral mucosa and a standardized amount of unstimulated saliva were plated on Chromagar ${ }^{\mathrm{TM}}$ Candida, and colony-forming units per millilitre were calculated. Initial speciation was based on colony color and was confirmed by standard germ tube, biotyping, or polymerase chain reaction assays.

Results-Infection with $C$. albicans was detected in seven transplant subjects and none of the controls. The transplant group had significantly higher oral Candida titers than the control group. There were no statistically significant relationships between the dose or type of immunosuppressants and oral Candida titers or infection. A significantly higher percentage of transplant subjects were colonized by more than one species, compared with control subjects. The most frequent species combination in transplant subjects was $C$. albicans and $C$. glabrata. C. glabrata was isolated from $13.5 \%$ of transplant carriers and none of the controls.

Conclusions-Increased oral Candida infection and carriage titers were found in the transplant population. Although the majority of transplant patients were colonized by $C$. albicans, $C$. glabrata appears to emerge as the second most prevalent species.

\section{Keywords}

Candida albicans; Candida glabrata; transplant; oral candidiasis

Oropharyngeal candidiasis (OPC) is an opportunistic infection caused by Candida, a ubiquitous fungal organism that is part of the normal microbiota of the oropharyngeal and

\footnotetext{
(C) 2009 John Wiley \& Sons A/S.

Anna Dongari-Bagtzoglou, Associate Professor and Chair, Division of Periodontology, School of Dental Medicine, University of Connecticut Health Center, 263 Farmington Avenue, Farmington, CT 06030-1710, USA Tel.: +1 860679 4543; fax: +1 8606791673 ; e-mail: E-mail: adongari@uchc.edu.
} 
gastrointestinal tract. OPC is a major cause of morbidity in immunocom-promised patients (7) and can predispose these patients to esophageal candidiasis $(19,46)$, an invasive form of infection with significant morbidity and higher risk for fatal, disseminated infection $(42,44)$. Candida albicans is the species implicated in the overwhelming majority of OPC cases in immunocompromised patients $(7,12)$, although other species such as Candida glabrata are recently emerging as possible etiological agents $(8,11)$.

Invasive fungal infections attributed to Candida species are particularly prevalent after organ transplantation, especially in the immediate post-transplant period (30). Candida can trigger bloodstream, esophageal, gastrointestinal, respiratory and uri-nary tract infections in solid organ transplant recipients, with prevalence during the first 6 months after transplantation ranging between 5 and $50 \%$ depending on the type of transplant (e.g. renal vs. liver) $(4,19$, 29). It was reported that up to $80 \%$ of Candida-esophagitis episodes in renal transplant patients were preceded by oral thrush or colonization $(4,19)$. In addition transplant patients with OPC, who are otherwise asymptomatic, are frequently discovered to have esophageal candidiasis upon endoscopy (21).

Despite the increased prevalence of other fungal infections in solid organ transplant recipients, the overall significant morbidity of OPC, and its possible implications in more invasive esophageal infections, few studies have assessed the prevalence of OPC in this population and all of these were conducted outside the USA $(3,12,18)$. In addition, the oral carriage frequency, intensity of carriage, and speciation of Candida colonizing transplant patients who are clinically stable, and consequently unlikely to present transient fluctuations in the oral microbial flora due to changes in the immunosuppressive regimen, have never been studied before. In this work we studied the prevalence of OPC, Candida carriage frequency, titers and species colonizing the oral mucosa, in clinically stable transplant patients who were at least 1 year post-transplant, and examined relevant risk factors.

\section{Materials and methods}

\section{Subject selection criteria}

One hundred and forty-four consecutive kidney and cardiac transplant patients were screened during routine, outpatient, follow-up visits at the transplant unit in Hartford Hospital, between March 2005 and 2006. We chose kidney and heart transplant recipients because these are the most commonly transplanted solid organs (Scientific registry of transplant recipients, http://www.ustransplant.org). Of these patients, 90 were included who met the following criteria: (i) at least 1 year post-transplant, (ii) no history of antibiotic, antifungal, or antiviral usage during the previous 4 months. All transplant subjects were clinically stable, which was defined as having stable serum creatinine levels compared with baseline (kidney only) and no signs of recurrent primary disease, or acute rejection (both organs). These patients were invited to enter the study after clearance by the physician following each case.

Control subjects included 72 age-matched individuals, recruited through an e-mail broadcast to University of Connecticut Health Center employees (36 subjects), local General Clinical Research Center and Women's Health Center mailing lists (16 subjects), or advertisements in local area newspapers ( 20 subjects), during the same time period. These subjects had no immunological compromising condition and had no history of antibiotic, antifungal, or antiviral use during the previous 4 months. After an explanation of the study, the overall riskbenefit analysis and final determination of eligibility, written informed consent was sought from each of the participants. Following the guidelines of the local Institutional Review Board, subjects were notified of their right to refuse to participate or to withdraw at any time, without affecting their medical or dental care. 


\section{Data collection}

The medical records of the transplant subjects were reviewed and all relevant information was collected using a standardized data extraction form. Information included subject demographics, medical history including history of fungal infections and antifungal medications, graft failure risk factors, history of acute or chronic graft rejection, and current medications.

\section{Oral examination and microbial sampling}

A complete oral examination was performed in all subjects. Oral colonization (healthy carrier state) was considered in asymptomatic, lesion-free subjects, with Candida-positive saliva or dorsal tongue swab cultures. Infection was defined as clinically suspicious lesions (erythematous, hyperplastic, or pseudomembranous) and testing positive by culture on BBL CHROMagar ${ }^{\mathrm{TM}}$ Candida medium (Becton Dickinson, Rockville, MD). The number of colonyforming units (CFU) of Candida present in a standardized amount of unstimulated saliva (3 $\mathrm{ml}$ ) was counted after plating samples on CHROMagar ${ }^{\mathrm{TM}}$ Candida medium and the CFU/ml of saliva was used as an indicator of Candida titer/load in each subject. Subjects were asked not to drink or eat for at least $1 \mathrm{~h}$ before saliva collection. Sterile oral swabs were additionally used to sample the dorsal surface of the tongue in all subjects, or the surface of clinically suspicious mucosal lesions in subjects with such lesions. Swab samples were plated directly on CHROMagar Candida medium and were used to complement species analyses. Colony number and color were assessed at $35^{\circ} \mathrm{C}$ for up to 5 days. Subjects with high Candida titers were considered those with $>1000 \mathrm{CFU} / \mathrm{ml}$ detected in unstimulated saliva $(15,25)$. Colonies were initially speciated by their characteristic color on CHROMagar Candida plates and confirmed by a germ tube test at $37^{\circ} \mathrm{C}$, and standard sugar assimilation assay profiles (RapID Yeast Plus System, Lenexa, KS). The vast majority of isolates were conclusively identified on the basis of the results of the RapID yeast identification system. Polymerase chain reaction was required to identify four of the $C$. glabrata isolates, which did not give conclusive sugar assimilation assay profiles. This selective polymerase chain reaction strategy for Candida speciation has been published by others in similar oral carriage epidemiological studies (23).

\section{Statistical analyses}

A logistic regression was run on the dichotomous dependent variables (Candida presence/ absence in saliva or tongue mucosa, and high/low Candida loads in saliva), as a function of the three-level predictor variable, i.e. transplant status (kidney, heart, or no transplant). $A$ priori contrasts were used that provided two orthogonal tests: either kidney vs. no transplant and heart vs. no transplant, or transplant vs. no transplant and kidney vs. heart. The results in these analyses are given as odds ratios (OR) together with 95\% confidence intervals (95\% CI). When a frequency of zero occurred within any of the three groups (kidney, heart, or no transplant), the Fisher's exact test was used to calculate significance levels. For continuous dependent measures (e.g. Candida in CFU/ml) a $t$-test or analysis of variance (for multiple group comparisons) was used to test whether groups had significantly different levels of the measure. Bivariate associations between continuous or dichotomous independent measures were run using the Pearson correlation test or the Yates-corrected chi-square test, respectively. Variables that were skewed were logarithmically transformed. Two-tailed tests were routinely used, and values in parentheses of respective tests indicate the degrees of freedom.

\section{Results \\ Population characteristics}

There were significantly more diabetics and former smokers in the transplant group compared with the control group (Table 1). There were only two current smokers in the transplant group. 
Two transplant subjects had a history of intra-oral (tongue) or peri-oral squamous cell carcinoma (lip), whereas three additional transplant subjects had a history of squamous or basal cell carcinoma of the skin in the neck and head regions. None of the controls had a history of head and neck neoplasia. This finding is consistent with the greater overall risk of transplant patients for developing skin or mucosal carcinomas $(13,24)$.

Overall, $23 \%$ of kidney and $33 \%$ of heart transplant recipients and none of the controls had a positive history of receiving oral antifungal treatment (Table 2). In the transplant group there was a significant association between the presence of diabetes and history of antifungal treatment (28\% of the diabetics in the transplant group had a positive history of antifungal use, compared to $10.3 \%$ of non-diabetics), such that those who had diabetes were more likely to

have received antifungals post-transplant, $\left(\chi_{(1)}^{2}=4.28, P=0.039\right.$, 2-t $)$. Subjects who had received antifungals were also on higher doses of prednisone, than those who did not receive oral antifungals $\left(t_{(87)}=2.54, P=0.013\right)$. In contrast, none of the transplant subjects on cyclosporine A treatment had a positive history of antifungal use, compared with $27.4 \%$ of those not on cyclosporine $\mathrm{A}$ who had been treated with antifungals. This resulted in a significant negative association between cyclosporine A and history of antifungal treatment $\left(\chi_{(1)}^{2}=8.84\right.$, $P=0.003,2-\mathrm{t})$.

\section{Oral clinical and microbiological findings}

There was no significant difference in the average number of missing teeth between the two groups or in the frequency of removable oral prostheses. The prevalence of self-reported xerostomia did not differ between the transplant and control groups $(P=0.4)$. Among the 90 transplant recipients, only seven (six kidney and one heart transplant subject) showed clinical and microbiological evidence of erythematous candidiasis on the dorsum of the tongue or hard palate, occasionally accompanied by a mild burning sensation. $C$. albicans was the species isolated from all these lesions. None of the controls had oral candidiasis, resulting in a significant difference from transplant subjects $(P=0.004)$.

The frequency of asymptomatic colonization in transplant recipients (57.7\%) was not significantly higher, compared with healthy controls (50\%). In addition, logistic regression showed that the type of transplant (heart vs. kidney) did not significantly affect the frequency of colonization (Wald $=0.08, P=0.78$, OR 1.22 , CI $0.31,4.87$ ). When all groups were considered, there was no relationship between the presence of diabetes and Candida colonization in the oral cavity $(P=0.57)$, or between the presence of diabetes and high $(>1000$ $\mathrm{CFU} / \mathrm{ml})$ vs. low ( $<1000 \mathrm{CFU} / \mathrm{ml})$, oral Candida titers $(P=0.4$ for all species, $P=0.19$ for $C$. albicans). Age $\left(r_{(160)}=0.11, P=0.15\right)$, and gender $\left(t_{(159)}=1.71, P=0.09\right)$, also did not significantly influence the total Candida titers in saliva.

The mean CFU/ml titers for all species were significantly higher in transplant subjects (mean $\log \mathrm{CFU} / \mathrm{ml}=18.4$ ) compared with controls (mean $\log \mathrm{CFU} / \mathrm{ml}=2.01, t_{(160)}=2.03, P=0.04$ ), whereas comparison of the mean log-transformed CFU/ml for C. albicans only, showed a trend for significance between the two groups (mean $\log \mathrm{CFU} / \mathrm{ml}$ for controls $=5.3$, mean log CFU/ $\mathrm{ml}$ for transplant $\left.12.2, t_{(160)}=1.66, P=0.09\right)$. When oral titers were compared between kidney and heart transplant subjects, there were no statistically significant differences in the mean log $\mathrm{CFU} / \mathrm{ml}$ titers for all species $\left(t_{(88)}=0.33, P=0.9\right)$ or for $C$. albicans $\left(t_{(88)}=0.56, P=0.5\right)$. Surprisingly, there was no statistically significant relationship between the presence of diabetes, type or dose of individual immunosuppressants, the presence of triple pharmacological immunosuppression, or the duration of immunosuppression, with oral Candida titers or infection. 
The frequency of C. albicans isolation in transplant and control carriers was similar (approximately $80 \%$, Fig. 1). However, the frequency of $C$. glabrata colonization was higher in the transplant group [8.6\% of all transplant subjects $(n=90)$, or $13.5 \%$ of transplant Candida carriers $(n=52)$ ], as compared with controls $(0 \%$ or $0 / 72$ healthy subjects,

$\chi_{(1)}^{2}=5.85, P=0.016,2-\mathrm{t}$, for subject-based comparisons). No other significant differences in the types of species isolated were noted between transplant and control carriers (Fig. 1), or between kidney and heart transplant subjects (Table 3). Seventeen of the $90(18.9 \%)$ transplant subjects (or $32.6 \%$ of the transplant Candida carriers) harbored multiple species in the oral cavity compared with three of $72(4.2 \%)$ healthy subjects (or $8.3 \%$ of healthy Candida carriers, $n=36$ ), a difference which was statistically significant $\left(\chi_{(1)}^{2}=4.00, P=0.045\right.$, 2-t, for subjectbased comparisons). The most frequent species combination in transplant subjects was $C$. albicans and C. glabrata.

\section{Discussion}

In this study we found a greater prevalence of OPC in subjects with functional renal or cardiac transplants later than 1 year post-transplant, compared with control subjects. Higher OPC prevalence has been previously reported in renal and liver transplant patients, but these reports included smaller or poorly characterized populations, and did not focus on patients who were clinically stable and at least 1 year post-transplant. Moreover, none of these previous studies included US populations $(3,6,9,14,18,39)$. Our findings suggest that the prevalence of this oral infection in solid organ transplant recipients in the US (7.7\% according to our study) may be lower compared with other areas in the word, such as the Middle East or eastern Europe, where reported prevalence rates range between 15 and $64 \%(3,14,18)$. Such differences may be attributable to variations in population characteristics among studies, including time after transplantation, immunosuppressant regimens or overall health status of the transplant populations examined. The OPC detected in our population was exclusively of the erythematous type and was fairly localized, which is consistent with other reports of OPC in renal transplant patients (3).

Immunosuppressant medications commonly taken by transplant recipients, such as corticosteroids, adversely affect all aspects of immunity (26) and certain antiproliferative medications, such as mycophenolate and azathioprine, can trigger neutropenia, which is one of the most important predisposing factors to candidiasis (7). The finding that phagocyte antifungal responses are significantly impaired in transplant recipients (32) may also help to explain their increased susceptibility to oroesophageal infection. Although immunosuppressants seemingly elevate the risk for OPC, significant associations between dosages and higher risk for infection were not demonstrated in this or other studies (3), possibly because of the requirement of pharmacologically homogeneous or larger transplant populations for statistically significant results.

Increased rates of asymptomatic oral colonization with Candida are common in immunosuppressed patients $(22,27,45)$. However, the oral Candida colonization rate in this US transplant population (57.7\%) was not significantly different from that in controls and was lower than that reported in transplant populations in other countries $(75-80 \%)(3,14)$. For reasons that are not entirely understood, geographic location is one of the determinant factors of both incidence of Candida infection and species distribution in population surveillance studies $(10,20,31)$. This may help to explain the disparities between our findings and the findings reported from other parts of the world.

Despite the reported differences in the epidemiology of fungal infections among different transplanted organs (30), we were not able to demonstrate significant differences in oral 
Candida colonization frequency, or titers between kidney and heart transplant subjects. This may be related to the fact that our heart transplant group is small, and consequently underpowered, for meaningful statistical comparisons. In addition, there were no differences in the clinical characteristics or immunosuppressive regimens between the two groups, which may explain the lack of significant differences in the oral microbiota. However, it should also be noted that the differences in the epidemiology of fungal infections among different organ transplants pertain mostly to the early post-transplant period and are related to environmental exposure of the transplanted organs (30).

Although oral colonization is one of the most frequently identified risk factors of OPC (16, $17,28,41)$, the clinical significance of the increased Candida titers in the oral cavity of transplant patients is unknown. A recent study in transplant patients showed that the vast majority of patients who developed invasive fungal infections were orally colonized with $C$. albicans and there was also a significant correlation between oral colonization and the subsequent development of invasive respiratory or gastrointestinal infection (4). Therefore, the increased oral fungal burden in this population may play a potentially important role in the subsequent development of infection. Although prophylactic antifungals have been shown to reduce intestinal colonization, they appear to be ineffective in lowering the incidence of oral mucosal infection or colonization in bone marrow transplant patients $(1,9,38,43)$. Therefore the routine prophylactic use of antifungals in transplant patients with high levels of oral Candida colonization is probably inappropriate, unless oral infection is present.

Although C. albicans was the primary oral Candida species isolate in this and other studies of immunocompromised patients $(22,45), C$. glabrata is isolated with increasing frequency in these patients $(2,40)$. Previously, $C$. glabrata was considered a relatively non-pathogenic saprophyte of the normal flora of healthy individuals $(8,11)$. In recent studies $C$. glabrata has emerged as a notable pathogenic agent in OPC in patients receiving radiotherapy (5,33-36). In fact, the failure of prophylactic antifungals in lowering the incidence of infection in immunocompromised patients has been partly attributed to the positive selective pressure of azoles on C. glabrata, which is naturally resistant, and the potential active role of this organism in infection (43). Although longitudinal studies are needed to determine the role of the increased oral C. glabrata carriage in the subsequent development of infection in transplant subjects, it has been reported that cancer patients who carried this yeast in saliva, throat, and rectum had the highest percentage of subsequent infection (2).

Mixed species of Candida were identified in a higher percentage of the transplant patients than in the control subjects in this study, a report that is in agreement with a previous study in a human immunodeficiency virus (HIV) -positive population (45). This finding may be indicative of the possibility that several species, which are normally more vulnerable to host immunity, are allowed to thrive under this immunosuppressed, more permissive host environment. The clinical significance of the presence of mixed Candida species in the oral cavity is not entirely understood. However, oral infections with mixed Candida species have been reported to be more persistent, and more difficult to treat (37). In addition, more severe symptoms have been noted in HIV-infected and cancer patients with mixed $C$. albicans and C. glabrata oral infections (33-35).

In conclusion, this study has shown an increased prevalence of oral infection and higher oral Candida titers in stable renal and cardiac transplant subjects, compared with healthy controls. The emergence of $C$. glabrata as a colonizer of the oral cavity in this population and the presence of multiple Candida species are also important novel findings in this study. Future longitudinal studies should explore the role of increased Candida carriage burden in the development of symptomatic oral or esophageal infection in this population. 


\section{Acknowledgments}

This study was supported by NIH/NIDCR grant R21DE16466 awarded to Dr Anna Dongari-Bagtzoglou and in part by a General Clinical Research Center grant from NIH (M01RR06192) awarded to the University of Connecticut Health Center. The authors would like to acknowledge the help of Drs N. Chang and D. Kao in oral clinical examinations and Mrs L. Kashleva for technical support in microbiological studies.

\section{References}

1. Aisner J, Murillo J, Schimpff SC, Steere AC. Invasive aspergillosis in acute leukemia: correlation with nose cultures and antibiotic use. Ann Intern Med 1979;90:4-9. [PubMed: 105657]

2. Aisner J, Schimpff SC, Sutherland JC, Young VM, Wiernik PH. Torulopsis glabrata infections in patients with cancer. Increasing incidence and relationship to colonization. Am J Med 1976;61:2328. [PubMed: 945692]

3. Al-Mohaya MA, Darwazeh A, Al-Khudair W. Oral fungal colonization and oral candidiasis in renal transplant patients: the relationship to Miswak use. Oral Surg Oral Med Oral Pathol Oral Radiol Endod 2002;93:455-460. [PubMed: 12029285]

4. Badiee P, Kordbacheh P, Alborzi A, Zeini F, Mirhendy H, Mahmoody M. Fungal infections in solid organ recipients. Exp Clin Transplant 2005;3:385-389. [PubMed: 16417449]

5. Belazi M, Velegraki A, Koussidou T, et al. Oral Candida isolates in patients undergoing radiotherapy for head and neck cancer: prevalence, azole susceptibility profiles and response to antifungal treatment. Oral Microbiol Immunol 2004;19:347-351. [PubMed: 15491459]

6. Diaz-Ortiz ML, Mico-Llorens JM, Gargallo-Albiol J, Baliellas-Comellas C, Berini-Aytes L, GayEscoda C. Dental health in liver transplant patients. Med Oral Patol Oral Cir Bucal 2005;10:66-72.

7. Dongari-Bagtzoglou A, Fidel PL Jr. The host cytokine responses and protective immunity in oropharyngeal candidiasis. J Dent Res 2005;84:966-977. [PubMed: 16246925]

8. Li L, Redding S, Dongari-Bagtzoglou AI. Candida glabrata, an emerging oral opportunistic pathogen. J Dent Res 2007;86:204-215. [PubMed: 17314251]

9. Elad S, Wexler A, Garfunkel AA, Shapira MY, Bitan M, Or R. Oral candidiasis prevention in transplantation patients: a comparative study. Clin Transplant 2006;20:318-324. [PubMed: 16824148]

10. Feld R. The role of surveillance cultures in patients likely to develop chemotherapy-induced mucositis. Support Care Cancer 1997;5:371-375. [PubMed: 9322348]

11. Fidel PL Jr, Vazquez JA, Sobel JD. Candida glabrata: review of epidemiology, pathogenesis, and clinical disease with comparison to C. albicans. Clin Microbiol Rev 1999;12:80-96. [PubMed: 9880475]

12. Fidel PL Jr. Distinct protective host defenses against oral and vaginal candidiasis. Med Mycol 2002;40:359-375. [PubMed: 12230215]

13. Glover M, Cerio R, Corbett M, Leigh I, Hanby AM. Cutaneous squamoproliferative lesions in renal transplant recipients. Differentiation from lesions in immunocompe-tent patients. Am J Dermatopathol 1995;17:551-554. [PubMed: 8599467]

14. Golecka M, Oldakowska-Jedynak U, Mierzwinska-Nastalska E, Adamczyk-Sosinska E. Candidaassociated denture stomatitis in patients after immunosuppression therapy. Transplant Proc 2006;38:155-556. [PubMed: 16504690]

15. Gottfredsson M, Cox GM, Indridason OS, de Almeida GM, Heald AE, Perfect JR. Association of plasma levels of human immunodeficiency virus type 1 RNA and oropharyngeal Candida colonization. J Infect Dis 1999;180:534-537. [PubMed: 10395877]

16. Grimoud AM, Lodter JP, Marty N, et al. Improved oral hygiene and Candida species colonization level in geriatric patients. Oral Dis 2005;11:163-169. [PubMed: 15888107]

17. Grimoud AM, Marty N, Bocquet H, Andrieu S, Lodter JP, Chabanon G. Colonization of the oral cavity by Candida species: risk factors in long-term geriatric care. J Oral Sci 2003;45:51-55. [PubMed: 12816366]

18. Gulec AT, Demirbilek M, Seckin D, et al. Superficial fungal infections in 102 renal transplant recipients: a case-control study. J Am Acad Dermatol 2003;49:187-192. [PubMed: 12894063] 
19. Gupta KL, Ghosh AK, Kochhar R, Jha V, Chakrabarti A, Sakhuja V. Esophageal candidiasis after renal transplantation: comparative study in patients on different immunosuppressive protocols. Am J Gastroenterol 1994;89:1062-1065. [PubMed: 8017366]

20. Hellstein J, Vawter-Hugart H, Fotos P, Schmid J, Soll DR. Genetic similarity and phenotypic diversity of commensal and pathogenic strains of Candida albicans isolated from the oral cavity. J Clin Microbiol 1993;31:3190-3199.

21. Kathuria P, Sakhuja V, Gupta KL, et al. Gastrointestinal complications after renal transplantation. 10 Year data from a North Indian Transplant Center. ASAIO J 1995;41:M698-M703. [PubMed: 8573895]

22. Lockhart SR, Joly S, Vargas K, Swails-Wenger J, Enger L, Soll DR. Natural defenses against Candida colonization breakdown in the oral cavities of the elderly. J Dent Res 1999;78:857-868. [PubMed: 10326730]

23. Davis AN, Brailsford S, Briadley K, Beighton D. Oral yeast carriage in patients with advanced cancer. Oral Microbiol Immunol 2002;17:79-84. [PubMed: 11929553]

24. Macarez R, Bossis S, Robinet A, Le Callonnec A, Charlin JF, Colin J. Conjunctival epithelial neoplasias in organ transplant patients receiving cyclosporine therapy. Cornea 1999;18:495-497. [PubMed: 10422866]

25. McCullough M, Jaber M, Barrett AW, Bain L, Speight PM, Porter SR. Oral yeast carriage correlates with presence of oral epithelial dysplasia. Oral Oncol 2002;38:391-393. [PubMed: 12076705]

26. Meuleman J, Katz P. The immunologic effects, kinetics, and use of glucocorticosteroids. Med Clin North Am 1985;69:805-816. [PubMed: 2997555]

27. Odds, FC. Candida and candidosis: a review and bibliography. Bailliere Tindall; London: 1988.

28. Oksala E. Factors predisposing to oral yeast infections. Acta Odontol Scand 1990;48:71-74. [PubMed: 2181813]

29. Patel R, Portela D, Badley AD, et al. Risk factor for invasive Candida and non-Candida fungal infections after liver transplantation. Transplantation 1996;62:926-934. [PubMed: 8878386]

30. Patterson JE. Epidemiology of fungal infections in solid organ transplant patients. Transpl Infect Dis 1999;1:229-236. [PubMed: 11428994]

31. Pfaller MA, Diekema DJ. Epidemiology of invasive candidiasis: a persistent public health problem. Clin Microbiol Rev 2007;20:133-163. [PubMed: 17223626]

32. Pursell K, Verral S, Daraiesh F, et al. Impaired phagocyte respiratory burst responses to opportunistic fungal pathogens in transplant recipients: in vitro effect of r-metHuG-CSF (Filgrastim). Transpl Infect Dis 2003;5:29-37. [PubMed: 12791072]

33. Redding SW, Kirkpatrick WR, Coco BJ, et al. Candida glabrata oropharyngeal candidiasis in patients receiving radiation treatment for head and neck cancer. J Clin Microbiol 2002;40:1879-1881. [PubMed: 11980984]

34. Redding SW, Kirkpatrick WR, Dib O, Fothergill AW, Rinaldi MG, Patterson TF. The epidemiology of non-albicans Candida in oropharyngeal candidiasis in HIV patients. Spec Care Dentist 2000;20:178-181. [PubMed: 11203894]

35. Redding SW, Marr KA, Kirkpatrick WR, Coco BJ, Patterson TF. Candida glabrata sepsis secondary to oral colonization in bone marrow transplantation. Med Mycol 2004;42:479-481. [PubMed: 15552650]

36. Redding SW, Zellars RC, Kirkpatrick WR, et al. Epidemiology of oropharyngeal Candida colonization and infection in patients receiving radiation for head and neck cancer. J Clin Microbiol 1999;37:3896-3900. [PubMed: 10565903]

37. Redding SW. Oral complications of cancer therapy. Tex Med 2003;99:54-57. [PubMed: 12772642]

38. Riley DK, Pavia AT, Beatty PG, Denton D, Carroll KC. Surveillance cultures in bone marrow transplant recipients: worthwhile or wasteful? Bone Marrow Transplant 1995;15:469-473. [PubMed: 7599574]

39. Romano C, Ghilardi A, Carcagni MR, De Aloe GB. A case of oral erosive candidosis in a kidney transplant patient. Mycoses 2004;47:524-526. [PubMed: 15601462]

40. Ruhnke M. Epidemiology of Candida albi-cans infections and role of non-Candida-albicans yeasts. Curr Drug Targets 2006;7:495-504. [PubMed: 16611037] 
41. Scully C, El-Kabir M, Samaranayake LP. Candida and oral candidosis: a review. Crit Rev Oral Biol Med 1994;5:125-157. [PubMed: 7858080]

42. Sheft DJ, Shrago G. Esophageal moniliasis. The spectrum of the disease. JAMA 1970;213:18591862. [PubMed: 5468912]

43. Shepp DH, Klosterman A, Siegel MS, Meyers JD. Comparative trial of ketoconazole and nystatin for prevention of fungal infection in neutropenic patients treated in a protective environment. J Infect Dis 1985;152:1257-1263. [PubMed: 3905986]

44. Thom K, Forrest G. Gastrointestinal infections in immunocompromised hosts. Curr Opin Gastroenterol 2006;22:18-23. [PubMed: 16319672]

45. Vargas KG, Joly S. Carriage frequency, intensity of carriage, and strains of oral yeast species vary in the progression to oral candidiasis in human immunodeficiency virus-positive individuals. J Clin Microbiol 2002;40:341-350. [PubMed: 11825940]

46. Walsh TJ, Gonzalez CE, Piscitelli S, et al. Correlation between in vitro and in vivo antifungal activities in experimental fluconazole-resistant oropharyngeal and esophageal candidiasis. J Clin Microbiol 2000;38:2369-2373. [PubMed: 10835005] 


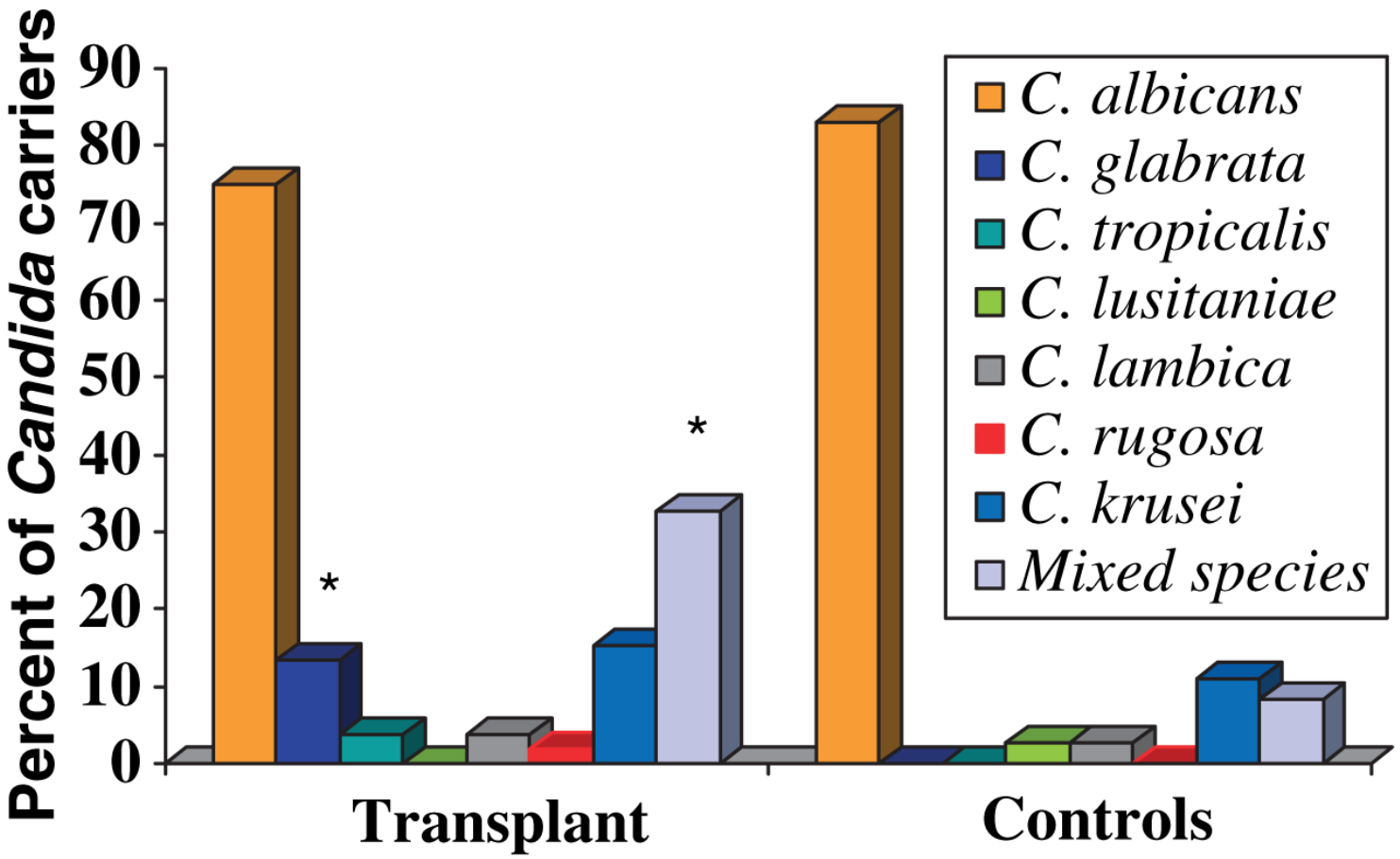

Fig. 1.

Frequency of Candida species isolated from the oral cavity of transplant and control Candida carriers. $* P<0.05$ compared to controls. 
Table 1

Summary of subject demographics

\begin{tabular}{|c|c|c|c|c|}
\hline Variable & Kidney $(n=81)$ & Heart $(n=9)$ & $\begin{array}{l}\text { Control subjects } \\
\quad(n=72)\end{array}$ & $\begin{array}{l}P \text { value, } \\
\text { transplant vs. } \\
\text { controls }\end{array}$ \\
\hline Age (years) & $52.7 \pm 11.9$ & $56.4 \pm 12.3$ & $53 \pm 10$ & 0.329 \\
\hline Gender (female) $(\%)$ & $42 \%$ & $44 \%$ & $60 \%$ & 0.03 \\
\hline Diabetics (\%) & $54 \%$ & $67 \%$ & $3 \%$ & $<0.0001$ \\
\hline Smokers (former) (\%) & $41 \%$ & $75 \%$ & $0 \%$ & $<0.0001$ \\
\hline Smokers (current) & $0 \%$ & $22 \%$ & $0 \%$ & 0.12 \\
\hline Removable dental prostheses & $3.7 \%$ & $0 \%$ & $0 \%$ & 0.11 \\
\hline Number of missing teeth & $4 \pm 4.6$ & $6.11 \pm 6.8$ & $1.69 \pm 2.1$ & 0.06 \\
\hline
\end{tabular}

Mean values and SD are shown for continuous variables and frequency values (\%) are shown for dichotomous variables. 
Table 2

Characteristics of transplant population

\begin{tabular}{llll}
\hline Clinical characteristics & Kidney $(\boldsymbol{n = 8 1})$ & Heart $(\boldsymbol{n}=\mathbf{9})$ & P value, kidney vs. heart \\
\hline Post-transplant years (mean) & $6.8 \pm 4.8$ & $8.1 \pm 5.3$ & 0.57 \\
Rejection history & $35 \%$ & $44 \%$ & 0.58 \\
History of antifungals & $23 \%$ & $33 \%$ & 0.50 \\
Cyclosporin & $31 \%$ & $33 \%$ & 0.88 \\
Prednisone & $93 \%$ & $89 \%$ & 0.70 \\
Mycophenolate & $74 \%$ & $89 \%$ & 0.33 \\
Tacrolimus & $63 \%$ & $56 \%$ & 0.67 \\
Azathioprine & $19 \%$ & $11 \%$ & 0.58 \\
Sirolimus & $11 \%$ & $11 \%$ & 1.00 \\
\end{tabular}

Mean values and SD are shown for number of years since transplant surgery, and frequency values (\%) are shown for dichotomous variables. 
Table 3

Frequency of Candida species isolation in each organ transplant type

\begin{tabular}{lccc}
\hline Candida species & Kidney $(\boldsymbol{n}=\mathbf{8 1})$ & Heart $(\boldsymbol{n}=\mathbf{9})$ & P value, kidney vs. heart \\
\hline C. albicans & $44 \%$ & $44 \%$ & 1.0 \\
C. glabrata & $8.6 \%$ & $0 \%$ & 0.36 \\
C. lambica & $2.4 \%$ & $11.1 \%$ & 0.17 \\
C. rugosa & $2.4 \%$ & $0 \%$ & 0.63 \\
C. krusei & $9.8 \%$ & $0 \%$ & 0.32 \\
C. tropicalis & $2.4 \%$ & $0 \%$ & 0.63 \\
$>1$ species & $19.7 \%$ & $11.1 \%$ & 0.53 \\
\hline
\end{tabular}

